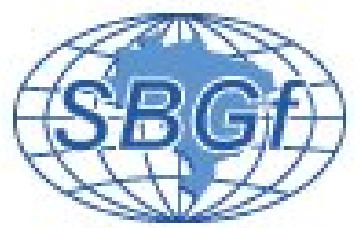

\title{
Dados Sísmicos com Grandes Afastamentos: Estudo de Caso com Modelos Sintéticos e Linha Sísmica do Campo de Tenerife, Colômbia
}

Francisco Gamboa Ortega (IGEO/UFBA), Amin Bassrei (IGEO/CPGG/UFBA), Ellen de Nazaré Souza Gomes (UFPA), Jessé Carvalho Costa (UFPA), Michelângelo Gomes da Silva (IGEO/UFBA).

Copyright 2012, SBGf - Sociedade Brasileira de Geofísica Este texto foi preparado para a apresentação no $V$ Simpósio Brasileiro de Geofísica, Salvador, 27 a 29 de novembro de 2012. Seu conteúdo foi revisado pelo Comitê Técnico do $V$ SimBGf, mas não necessariamente representa a opinião da SBGf ou de seus associados. É proibida a reprodução total ou parcial deste material para propósitos comerciais sem prévia autorização da SBGf.

\section{RESUMO}

O processamento sísmico de dados de reflexão convencional para obteção de imagens da sub-superfície inclui um fator de correção mediante a equação do tempo de propagação da onda (fonte - refletor - receptor), sendo que esta equação representa uma hipérbole para um meio isotrópico. Um modelo matemático mais próximo da realidade é um meio transversalmente isotrópico (ou VTI), e que pode ser representado com dois parâmetros: a velocidade NMO $\left(V_{n m o}\right)$ e o parâmetro de anelipsidade $(\eta)$ associado à uma curva não hiperbólica na equação do tempo de propagação. A interpretação sísmica destas imagens em meios geologicamente complexos é geralmente uma tarefa complicada, uma vez que esses meios se comportam como meios anisotrópicos, mas são processados ignorando a anisotropia. Neste trabalho é revista a metodologia para a análise de velocidade considerando dados sísmicos adquiridos em meios com anisotropia. Depois, para um modelo sintético, é feita uma comparação na análise de velocidade considerando um meio com anisotropia e outro sem anisotropia. Por fim, a metodologia para a medida de anisotropia é aplicada a um dado real 2D terrestre do Campo de Tenerife na Colômbia.

\section{INTRODUÇÃO}

A interpretação sísmica de imagens de meios geologicamente complexos é geralmente uma tarefa complicada. Um dos fatores que contribui para essa dificultade é o processamento realizado nesses dados. Normalmente no processamento de dados sísmicos a anisotropia é ignorada, entretanto, meios geologicamente complexos em regime quase estático comportam-se efetivamente como meios anisotrópicos (Helbig, 1994).

Estudos têm demonstrado que a presença de anisotro- pia sísmica do tipo VTI produz distorções significativas na análise convencional de dados sísmicos, como por exemplo, o fato que a velocidade NMO não é igual a velocidade RMS na condição de grandes e também pequenos afastamentos. Este meio produz um moveout não hiperbólico que se manifesta de forma signicativa em grandes afastamentos para o caso das ondas $P P$. Já para o caso das ondas $P S$ essa curva é observada em todos os afastamentos sejam grandes ou pequenos (Alkhalifah, 1997). Uma solução para este problema é remover os traços sobrecorregidos e empilhar os outros obtendo-se assim uma imagem que não proporciona a informação mais completa (Calderón, 2003).

A representação matemática do tempo de viagem da energia ao receptor com uma hipérbole deslocada exibe uma melhoria significativa na correção de sobretempo normal NMO para dados sísmicos. Esta aproximação requer três parâmetros que são:

- O tempo de viagem fonte - refletor - receptor é igual ao tempo de tempo de afastamento nulo.

- A velocidade normal moveout.

- O momento ponderado do tempo, que é designado por $\mu_{j}$.

O objetivo da hipérbole deslocada é aumentar a resolução que não atinge a equação convencional em dados sísmicos através do aumento de coerência (Castle, 1990).

Este trabalho tem por objetivo analisar através da metodologia apresentada em Alkalifah e Tsvankin (1995), a presença de anisotropia em um dado real terrestre 2D cujo lanço é de 9.000 metros, dado esse adquirido no Campo de Tenerife, Colômbia. Inicialmente, a metodologia apresentada em Alkalifah e Tsvankin (1995) é aplicada a um dado sintético com a mesma geometria de aquisição da linha real. Posteriormente é aplicada com a metodologia apresentada por (Castle, 1990).

\section{METODOLOGIA}

O método convencional para análise de velocidade no processamento de dados sísmicos supõem um refletor 
ideal, isto é, uma interface plana, homogênea, com espessura constante, sem perda de energia e num meio não-dispersivo. A representação matemática do tempo de viagem da fonte ao receptor é dada por (Dix, 1995)

$$
t_{x}^{2}=t_{0}^{2}+\frac{x^{2}}{V_{n m o}^{2}}
$$

Nessa curva de caráter hiperbólico, a variável $t_{0}$ é o tempo de chegada vertical (afastamento zero), $x$ é a distância fonte-receptor e $V_{n m o}$ é a velocidade moveout normal. Na análise de velocidade do método convencional é assumido que $V_{n m o}=V_{r m s}$. A equação NMO padrão utilizada pela indústria considera apenas os dois primeiros termos na expansão da série de Taylor (ver equação (1)). Porém, este tratamento é adequado apenas para pequenos afastamentos. Para um meio verticalmente isotrópico (ou $\mathrm{VTI}$ ) é preciso a utilização de mais termos na expansão da série de Taylor para uma correção NMO adequada, tal como utilizado por Alkhalifah (1995):

$$
t_{x}^{2}=t_{0}^{2}+\frac{x^{2}}{V_{n m o}^{2}}-\frac{2 \eta x^{4}}{V_{n m o}^{2}\left[t_{0}^{2} V_{n m o}^{2}+(1+2 \eta) x^{2}\right]} .
$$

A equação (2) mostra um terceiro termo em na expansão da série de Taylor que inclui um novo parâmetro designado por $\eta$, que tal como a velocidade $V_{n m o}$ é uma incógnita (Alkhalifah, 1995).

A Figura 1 mostra o tempo de viagem da fonte ao receptor para um modelo de uma camada plana utilizado a equação (2) e sua respectiva correção NMO para três valores de $\eta$, sendo que a curva azul tem um valor de $\eta_{1}=0$, a curva verde tem um valor de $\eta_{2}=0.1$ e a curva vermelha tem um valor de $\eta_{3}=0.2$. Note que

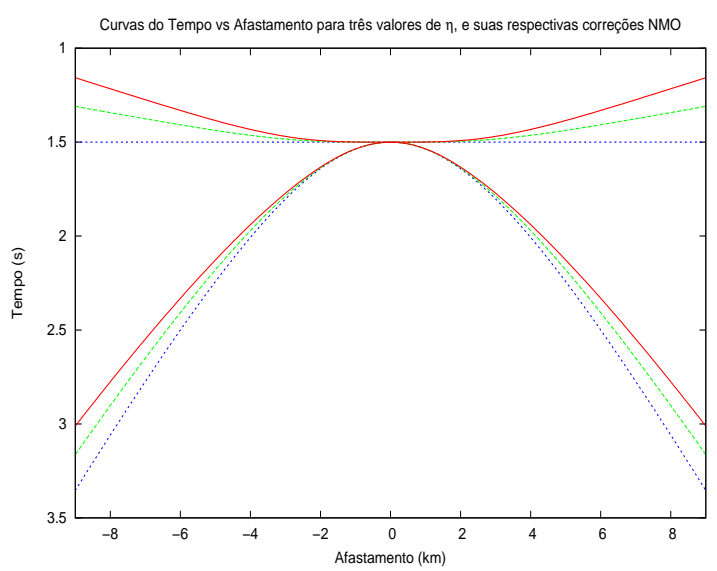

Figura 1: Tempo de viagem da fonte ao receptor para um modelo com um refletor horizontal a uma profundidade de $2.25 \mathrm{~km}$ com uma $V_{n m o}=3.0 \mathrm{~m} / \mathrm{s}$ (curvas inferiores) e a sua respectiva correção NMO para três valores de $\eta$ em um meio VTI (curvas superiores). na Figura 1, para afastamentos maiores de $4 \mathrm{~km}$ a partir do ápice da hipérbole, a correção NMO se apresenta sobrecorregida. Para valores de $\eta_{1}=0$ a equação (2) muda para a equação (1).

\section{Análise de velocidade método de Alkhalifah (1997)}

Para grandes afastamentos em um meio VTI a equação (2) têm dois paramentos desconhecidos: $V_{n m o}$ e $\eta$. A busca desses parâmetros está dividida em dois passos. No primeiro passo usa-se o arquivo de velocidades obtido a partir de uma análise de velocidade convencional, que é eficaz para afastamentos relativamente pequenos. No segundo passo usa-se a velocidade obtida do primeiro passo e o arquivo com os valores do parâmetro $\eta$, obtido mediante uma analise de semblance tipo coerência (Alkhalifah, 1995). Finalmente é realizada a correção NMO com esses dois arquivos.

A metodologia implementada neste trabalho propõe inicialmente a sequência convencional de processamento sísmico dos dados, incluindo uma análise de velocidade para grandes afastamentos, ou seja, a execução da correção NMO incluindo mais de dois termos na expansão da série de Taylor, a fim de se comparar estes resultados sintéticos com o resultado do processamento do dado reais.

Utilizamos um modelo de anisotropia VTI com cinco camadas, cuja relação afastamento-profundidade é maior do 1,5 , no caso $9 \mathrm{~km}$ de afastamento e $5 \mathrm{~km}$ de profundidade. 0 modelo escolhido não apresenta anisotropia nas camadas 1 e 5 . Os parâmetros de cada camada do modelo são mostrados na tabela 1 .

Os valores de $\epsilon$ e $\delta$ da Tabela 1 são nulos num meio isotrópico e o valor de $\gamma$ (Thomsen, 1986) corresponde a zero, porque assumimos a propagação da onda no plano $x z$. O parâmetro $\eta$ é definido como:

$$
\eta=\frac{\epsilon-\delta}{1+2 \delta}
$$

que é a aproximação anisotrópica de anelipsidade introduzida por Alkhalifah e Tsavankin (1995). A velocidade de grupo da onda $P$ em um meio anisotrópico $\mathrm{VTI}$ pode ser expressa em função do ângulo:

$$
v_{p}^{2}=v_{p 0}\left(1+\delta \operatorname{sen}^{2} \theta+2 \eta \operatorname{sen}^{4} \theta\right),
$$

onde $v_{p 0}=v_{p}(0)$ é a velocidade vertical. A velocidade na direção horizontal é expressa por

$$
v_{x}^{2}=v_{p}^{2}(\theta=\pi / 2)=v_{p 0}^{2}(1+2 \epsilon) .
$$

Outra quantidade importante é a velocidade $V_{n m o}$, definida como:

$$
V_{n m o}^{2}=v_{p 0}^{2}(1+2 \delta) \text {. }
$$




\begin{tabular}{|l|c|c|c|c|c|c|c|}
\hline & $\rho\left(\mathrm{g} / \mathrm{cm}^{3}\right)$ & $\epsilon$ & $\delta$ & $\gamma$ & $\eta$ & $V_{p} \mathrm{~m} / \mathrm{s}$ & $V_{s} \mathrm{~m} / \mathrm{s}$ \\
\hline Camada 1 & 2,16 & 0 & 0 & 0 & 0 & 2000 & 1400 \\
\hline Camada 2 & 2,23 & 0,081 & $-0,178$ & 0 & 0,39 & 2409 & 1324 \\
\hline Camada 3 & 2,29 & 0,2182 & 0,028 & 0 & 0,18 & 2757 & 1509 \\
\hline Camada 4 & 2,38 & 0,512 & 0,242 & 0 & 0,15 & 3308 & 2260 \\
\hline Camada 5 & 2,57 & 0 & 0 & 0 & 0 & 4450 & 2300 \\
\hline
\end{tabular}

Tabela 1: Parâmetros anisotrópicos do modelo.

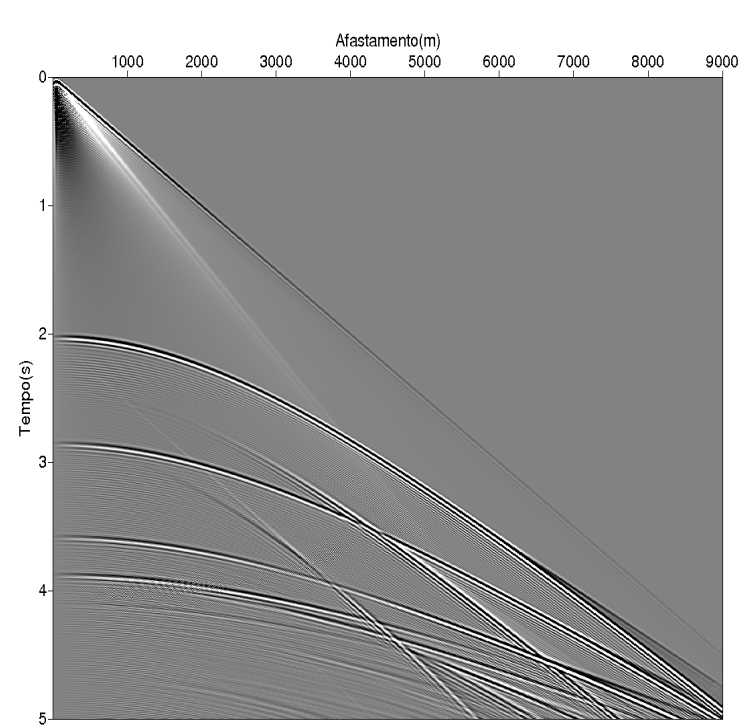

Figura 2: Sismograma ilustrando modelo anisotrópico com os eventos interpretados.

A Figura 2 mostra o sismograma referente ao modelo para um meio VTI homogêneo com as reflexões das ondas longitudinais $(P P)$ para as quatro interfaces. Para afastamentos a partir de $3.000 \mathrm{~m}$ pode-se ver as ondas convertidas $(P S)$ para a primeira e segunda interfaces do modelo. Os parâmetros anisotrópicos estão indicados na Tabela 1. A Figura 3 mostra a correção NMO e a respectiva análise de velocidade hiperbólica para o CDP 790, que é um CDP com grande afastamento. O procedimento anterior permite encontrar o arquivo das velocidades NMO, e após isso deve-se obter o arquivo que contêm os parâmetros $\eta$, mediante uma análise tipo semblance, conforme ilustrado na Figura 4.

Com os arquivos das velocidades e dos valores de $\eta$ foi efetuado o empilhamento dos dados, cujo resultado é mostrado na Figura 5. Note-se que nessa mesma Figura estamos mostrando também o resultado do empiIhamento convencional.

\section{ANÁLISE DE VELOCIDADE PELO MÉTODO DE CASTLE (1994)}

A representação matemática do tempo de viagem da fonte ao receptor com uma hipérbole deslocada exibe
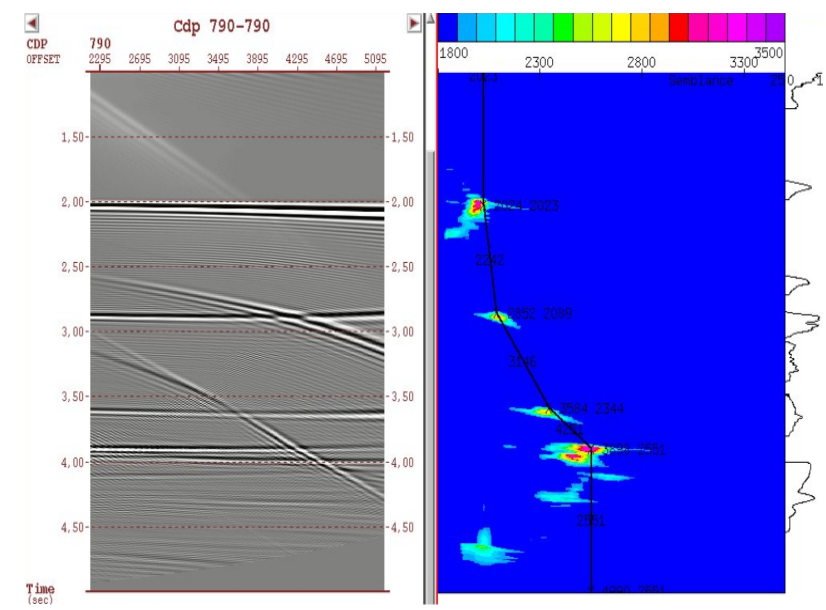

Figura 3: Correção NMO e a análise de velocidade respectivamente, com aproximação hiperbólica para um CDP com grande afastamento.
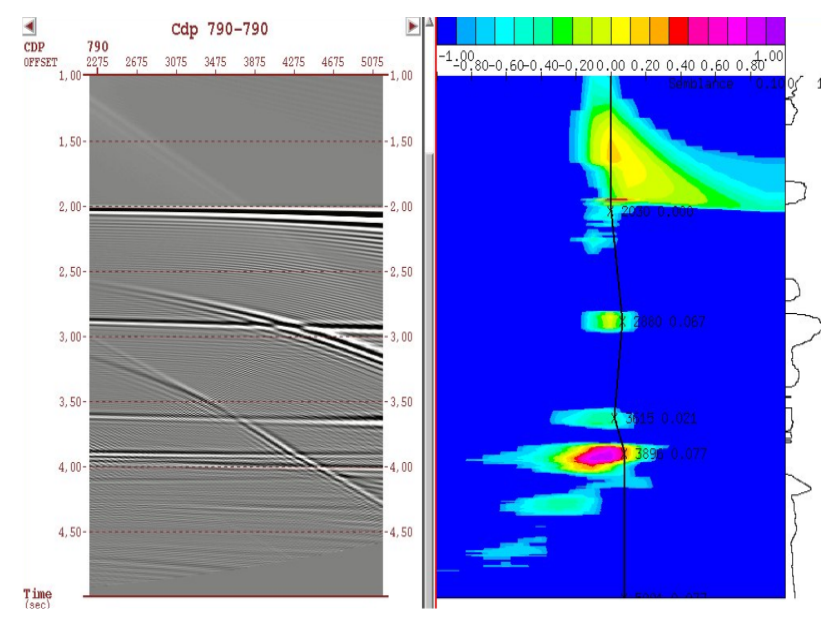

Figura 4: Correção NMO utilizando a aproximação não hiperbólica e análise do parâmetro $\eta$ tipo semblance com a para o CDP 790.

uma melhoria significativa na correção de sobretempo normal NMO para dados sísmicos ou aumento de coerência (Castle, 1997). Uma expressão matemática mais exata para a equação de sobretempo normal é dada por Malovichko (1978): 


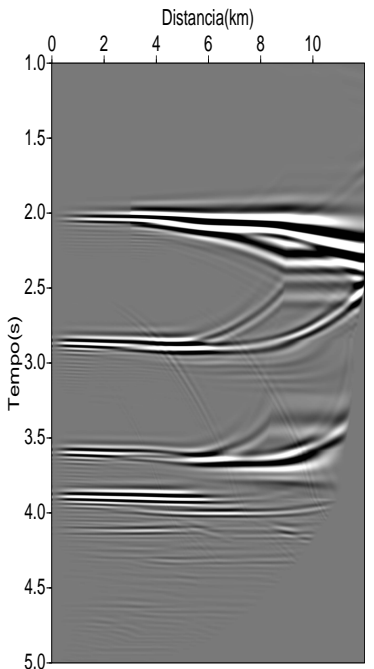

(a) (b)

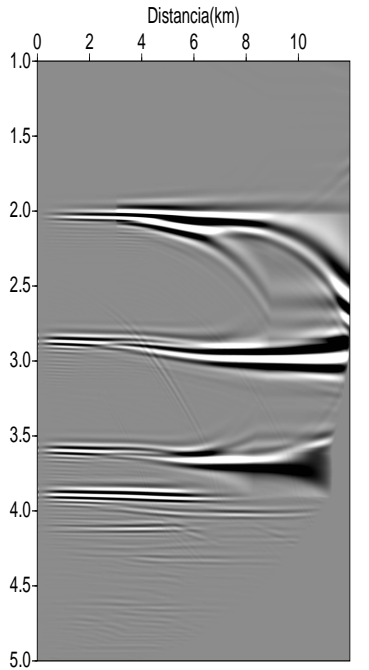

Figura 5: (a) Empilhamento dos dados após correção de NMO hiperbólica. (c) Empilhamento dos dados após correção de NMO não hiperbólica.

$$
t=\tau_{s}+\sqrt{\tau_{0}^{2}+\frac{x^{2}}{\nu^{2}}},
$$

onde $\tau_{0}$ é expresso como

$$
\tau_{0}=\frac{t_{0}}{S}
$$

e $\tau_{s}$ é o tempo de interseção das assíntotas da hipérbola da equação de Dix com o eixo do tempo:

$$
\tau_{s}=\tau_{0}(S-1)
$$

Ainda nas equações acima $t_{0}$ é o tempo de chegada vertical e $v$ é a velocidade interavalar de modo que

$$
v^{2}=S V_{r m s}^{2}
$$

e o fator $S$ é expresso

$$
S=\frac{\mu_{4}}{\mu_{2}^{2}}
$$

O valor de $\mu_{j}$, já apresentado como o momento ponderado do tempo é expresso por:

$$
\mu_{j}=\frac{\sum_{k=1}^{N} \Delta \tau_{k} V_{k}^{j}}{\sum_{k=1}^{N} \Delta \tau_{k}},
$$

sendo $V_{k}$ a velocidade intervar da $k$-ésima camada e $\Delta \tau_{k}$ o tempo vertical da $k$-ésima camada. Utilizamos um segundo modelo com anisotropia VTI e quatro camadas, cuja relação afastamento-profundidade é igual a 2,0 . Neste modelo a camada 1 não apresentam anisotropia. Os parâmetros de cada camada do modelo são mostrados na tabela 2 .
Para observar melhor o efeito de anisotropia houve um aumento no valor do parâmetro $\eta$ para as camadas 2 e 3 , sendo que a camada 4 foi suprimida. Os resultados são mostrados na Figura 6, onde também os principais eventos são identificados por pequenas setas vermelhas.

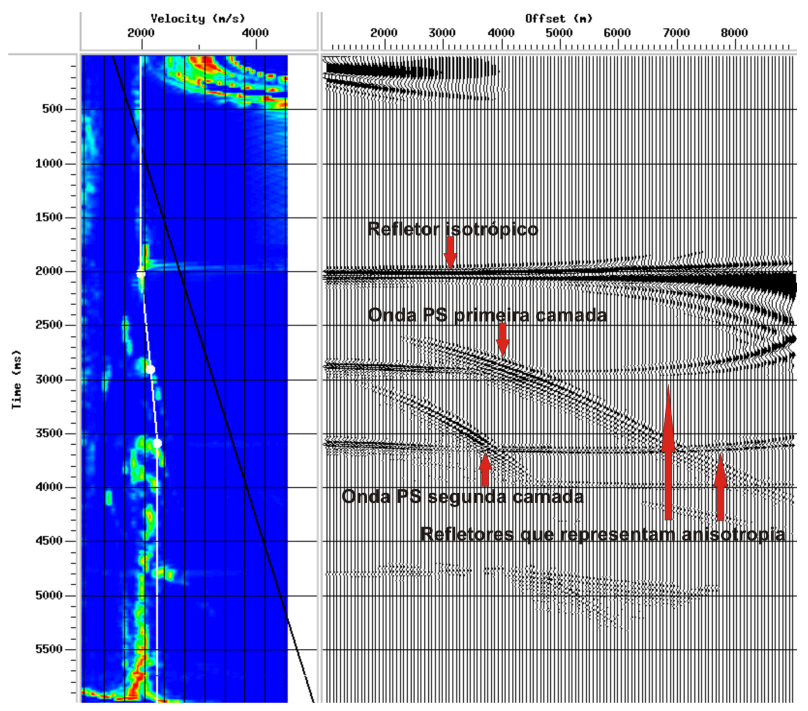

Figura 6: Correção NMO utilizando a aproximação hiperbólica ou equação de Dix.

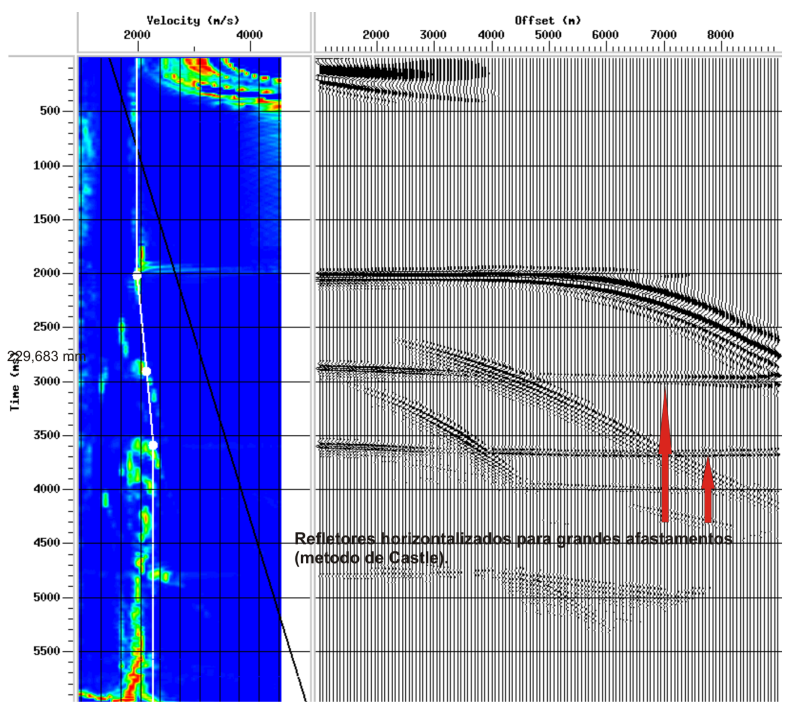

Figura 7: Correção NMO utilizando a aproximação não hiperbólica (método de Castle, 1994).

A comparação entre uma correção NMO convencional e uma hipérbole deslocada são mostrados na Figura 6 e 7. Para fazer uma correção na curva residual, aplicamos o método do Castel (1994) que utiliza a equação de Malovichko (1978). Pode-se notar a diferença entre a correção NMO convencional e a correção de Castle (1994) nos afastamentos de 7000 a $9000 \mathrm{~m}$ conforme indicado pelas duas setas vermelhas em ambas as Figuras. Esse resultado é devido ao aumento da coerência 


\begin{tabular}{|l|c|c|c|c|c|c|c|}
\hline & $\rho\left(\mathrm{g} / \mathrm{cm}^{3}\right)$ & $\epsilon$ & $\delta$ & $\gamma$ & $\eta$ & $V_{p} m / s$ & $V_{s} \mathrm{~m} / \mathrm{s}$ \\
\hline Camada 1 & 2,16 & 0 & 0 & 0 & 0 & 2000 & 1400 \\
\hline Camada 2 & 2,23 & 0,081 & $-0,174$ & 0 & 0,39 & 2409 & 1324 \\
\hline Camada 3 & 2,29 & 0,081 & -0.174 & 0 & 0,39 & 2757 & 1509 \\
\hline Camada 4 & 2,38 & 0,081 & -0.174 & 0 & 0,39 & 3308 & 2260 \\
\hline
\end{tabular}

Tabela 2: Parâmetros anisotrópicos do modelo para o método de Castle (1994).

com a equação de Malovichko (1978).

\section{RESULTADOS COM DADOS REAIS}

Para analisar a possibilidade de se detectar anisotropia, utilizamos nesse trabalho uma linha sísmica 2D terrestre cujo lanço é de $9000 \mathrm{~m}$. Tal linha foi adquirida no Campo de Tenerife, que está localizado no Valle Médio Del Magdalena (VMM). Por seu tuo, o VMM está localizado entre as serras Central e Oriente, a cerca de 260 $\mathrm{km}$ da direção norte-nordeste de Bogotá, Colômbia. O VMM é limitado ao norte pela falha de Bucaramanga e cobre uma área de $28.300 \mathrm{~km}^{2}$.

Os dados utilizados no processamento sísmico foram adquiros em 2010, e são de posse da Ecopetrol (Empresa Colombiana de Petróleo). Os dados correspondem a uma linha sísmica multicomponente 2D, obtida através do levantamento contínuo/sequencial, empregando a técnica CDP e usando o arranjo assimétrico.

A execução do levantamento se caracteriza por deslocamentos sucessivos da fonte no lanço que é mantido fixo. A fonte é deslocada ao longo de uma direção mais ou menos retilínea. Os acelerômetros estão distanciados de $10 \mathrm{~m}$ e as estações de tiro distam de $40 \mathrm{~m}$.

\section{Análise de velocidade para a Linha de Tenerife}

O mesmo procedimento para o processamento sísmico dos modelos sintéticos foi aplicado à linha de Tenerife. A Figura 8 utiliza a aproximação convencional hiperbólica para a análise de velocidade no supergather na faixa 982-1064 e CDP central número 1023, que é um CDP com grande afastamento.

Conforme afirmando na Introdução, o objetivo principal do trabalho é fazer uma comparação entre os resultados do método convecional e o que utiliza uma análise de velocidade não-hiperbólica, sendo que este último inclui um fator de anisotropia na construção do espectro de velocidades.

Depois de uma correção NMO utilizando a aproximação hiperbólica para o supergather na faixa 982-1064 e CDP central número 1023, verificamos que a curva residual esperada para os deslocamentos distantes em um meio

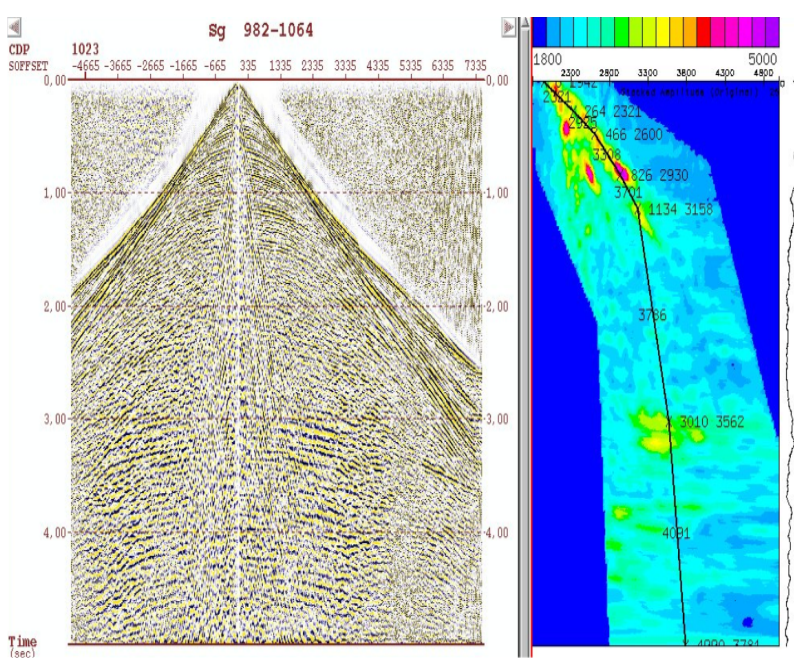

Figura 8: Análise de velocidade utilizando a aproximação hiperbólica para o supergather na faixa 982-1064 e CDP central número 1023, que é um CDP com grande afastamento.

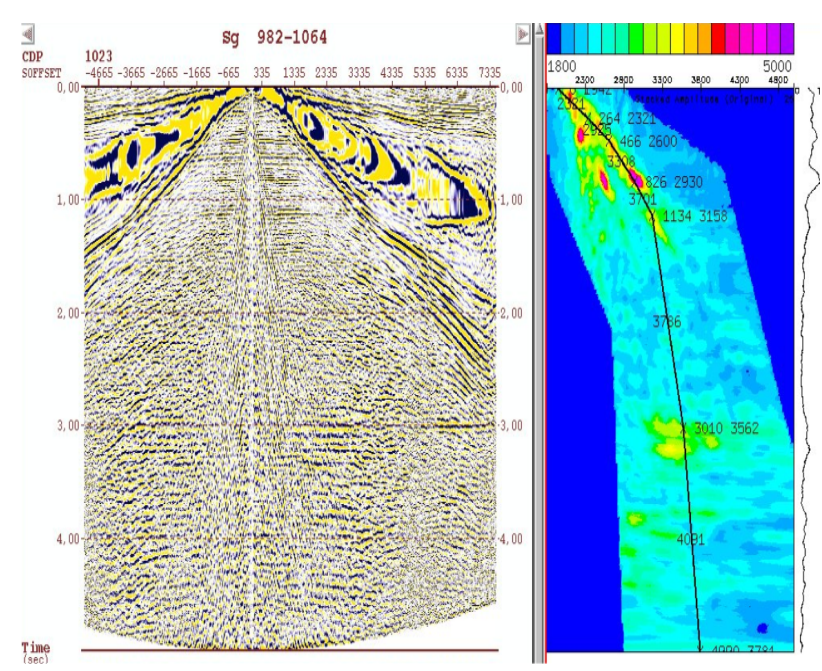

Figura 9: Correção NMO utilizando a aproximação hiperbólica para o supergather na faixa 982-1064 e CDP central número 1023.

anisotrópico VTI não foi observada nesse conjunto de dados. Portanto, o procedimento para determinar o valor do arquivo $\eta$ mostrou-se desnecessário, já que uma correção NMO hiperbólica (ver Figura 9) horizontalizou 
as reflexões de forma satisfatória.

\section{CONCLUSÕES}

Para uma relação afastamento-profundidade menor que um $(x / z<1,0)$, não se observa o efeito da anisotropia na posição de geofones distantes, de modo que a aplicação da correção NMO usando a aproximação hiperbólica irá horizontalizar as reflexões de forma satisfatória. Para grandes afastamentos a correção NMO convencional, isto é, hiperbólica, resulta numa curva residual nas camadas anisótropicas, para os eventos de grande afastamento e pouca profundidade. A velocidade NMO não é igual a velocidade RMS na presença de afastamentos próximos e distantes em um meio VTI. Para um meio anisótropico se produz uma reflexão na forma não hiperbólica proveniente de uma interface, que se manifesta de forma significativa em afastamentos distantes, para o caso das reflexões de ondas PP. Para o caso das reflexões de ondas $P S$ as reflexões de forma não hiperbólica são observadas em todos os afastamentos, sejam próximos ou distantes.

Como resultado da correção NMO ocorrem distorções nas frequências especialmente para os eventos mais rasos e de grande afastamento. Estas distorções são chamadas de estiramento, e no processamento convencional o efeito do estiramento é removido através do muting de um percentual da parte afetada, e uma correção não hiperbólica pode corrigir este estirameinto sem apagar informações de afastamentos distantes. A curva residual esperada para os deslocamentos distantes em um meio anisotrópico não é observada nos dados da linha de Tenerife, de modo que o procedimento para determinar o valor do arquivo $\eta$ mostrou-se desnecessário, já que uma correção NMO hiperbólica irá horizontalizar as reflexões de forma satisfatória.

\section{AGRADECIMENTOS}

Os autores agradecem à ANP (Projeto Campo EscolaUFBA), à CAPES, ao CNPq (INCT-Geofísica de Petróleo), à FINEP (Rede CT-PETRO em Geofísica de Exploração) pelo financiamento e apoio à pesquisa. Também agradecem às empresas Landmark e Paradigm pelos acordos de cooperação que permitem o uso das licenças dos softwares de processamento e interpretação sísmica disponibilizadas ao Laboratorio de Geofisica de Exploração de Petróleo do CPGG-UFBA. Francisco Gamboa Ortega agradece à CAPES pela bolsa de mestrado, através do INCT-GP. Amin Bassrei e Jessé Carvalho Costa agradecem ao CNPq pela bolsa de produtividade de pesquisa.

\section{REFERÊNCIAS BIBLIOGRÁFICAS}

Alkhalifah, T., and Tsvankin, I., 1995. Velocity analysis for transversely isotropic media: Geophysics, 60: 15501566.

Alkhalifah, T., 1997. Velocity analysis using nonhyperbolic moveout in transversely isotropic media: Geophysics, 62: 1839-1854.

Castle R.J. 1994. A theory of normal moveout: Geophysics, 59: 983-999.

Calderón, C., 2003. Procesamiento en tiempo de datos sísmicos de reflexión de ondas $\mathrm{P}$ en medios con anisotropía transversal con eje de simetría vertical (VTI). Trabalho final de curso. Universidad Simon Bolivar, Venezuela.

Dix, C. H.,1955. Seismic velocities from surface measurements: Geophysics, 20: 68-86.

Helbig, K., 1994. Foundation of anisotropy for exploration seismics. Pergamon, Oxford, 486 pp.

Malovichko, A. A., 1978. A new representation of the traveltime curve of reflected waves in horizontally layered media: Applied Geophysics, 91, 47-53.

Thomsen, L., 1986. Weak elastic anisotropy: Geophysics, 51: 1954-1966.

Tsvankin, I., and Thomsen, L., 1994. Nonhyperbolic reflection moveout in anisotropic media: Geophysics, 59: 1290-1304. 